Research paper

\title{
Association between harm reduction intervention uptake and recent hepatitis $C$ infection among people who inject drugs attending sites that provide sterile injecting equipment in Scotland
}

\author{
Elizabeth J. Allen ${ }^{\mathrm{a}}$, Norah E. Palmateer ${ }^{\mathrm{b}, *}$, Sharon J. Hutchinson ${ }^{\mathrm{b}, \mathrm{c}}$, Sheila Cameron ${ }^{\mathrm{d}}$, \\ David J. Goldberg ${ }^{\mathrm{b}}$, Avril Taylor ${ }^{\mathrm{a}}$ \\ a Institute for Applied Social and Health Research, School of Social Sciences, University of the West of Scotland, Paisley Campus, Paisley PA1 2BE, United Kingdom \\ ${ }^{\mathrm{b}}$ Health Protection Scotland, 4th Floor Meridian Court, 5 Cadogan Street, Glasgow G2 6QE, United Kingdom \\ ${ }^{c}$ Department of Mathematics and Statistics, University of Strathclyde, 26 Richmond Street, Glasgow G1 1XH, United Kingdom \\ ${ }^{\mathrm{d}}$ West of Scotland Specialist Virology Centre, Gartnavel General Hospital, Glasgow G12 0YN, United Kingdom
}

\section{A R T I C L E I N F O}

\section{Article history:}

Received 12 March 2012

Received in revised form 6 July 2012

Accepted 16 July 2012

\section{Keywords:}

Hepatitis $C$ virus

Injection equipment

Methadone

Recent infection

\begin{abstract}
A B S T R A C T
Background: Prevalence of the hepatitis C virus (HCV) among people who inject drugs (PWID) in Scotland is high. The Scottish Government has invested significantly in harm reduction interventions with the goal of reducing HCV transmission among PWID. In evaluating the effectiveness of interventions, estimates of HCV incidence are essential.

Methods: During 2008-2009, PWID were recruited from services providing sterile injecting equipment across mainland Scotland, completed an interviewer-administered questionnaire and provided a dried blood spot for anonymous anti-HCV and HCV-RNA testing. Recent infections were defined as anti-HCV negative and HCV-RNA positive. Logistic regression was undertaken to examine associations between recent HCV infection and self-reported uptake of methadone maintenance therapy (MMT) and injection equipment.

Results: Fifty-four percent (1367/2555) of participants were anti-HCV positive. We detected 24 recent HCV infections, yielding incidence rate estimates ranging from 10.8 to 21.9 per 100 person-years. After adjustment for confounders, those with high needle/syringe coverage had reduced odds of recent infection (adjusted odds ratio [AOR] 0.32, 95\% CI 0.10-1.00, p=0.050). In the Greater Glasgow \& Clyde region only, we observed a reduced odds of recent infection among those currently receiving MMT, relative to those on MMT in the last six months but not currently (AOR 0.04, 95\% CI 0.001-1.07, p=0.055). The effect of combined uptake of MMT and high needle/syringe coverage was only significant in unadjusted analyses (OR 0.34, 95\% CI 0.12-0.97, $p=0.043$; AOR 0.48, 95\% CI 0.16-1.48, p=0.203).

Conclusion: We report the first large-scale, national application of a novel method designed to determine incidence of HCV among PWID using a cross-sectional design. Subsequent sweeps of this survey will increase statistical power and allow us to gauge the impact of preventive interventions.
\end{abstract}

(c) 2012 Elsevier B.V. All rights reserved.

\section{Introduction}

Risk behaviour associated with injecting drug use is the main mode of transmission of the hepatitis $\mathrm{C}$ virus (HCV) in resourcerich countries. In Scotland, prevalence rates of $21-70 \%$ have been detected among regional populations of people who inject drugs (PWID) in recent years (University of the West of Scotland, 2010). Recognising the importance of tackling the HCV epidemic, the Scottish Government launched the Hepatitis C Action Plan for Scotland, which invested in the development of services to prevent,

\footnotetext{
* Corresponding author. Tel.: +44 141300 1416; fax: +44 1413001170.

E-mail address: norah.palmateer@nhs.net (N.E. Palmateer).
}

diagnose, treat and care for individuals who are infected with HCV, or who are at risk of acquiring HCV infection (Scottish Government, 2008). In relation to prevention, the Action Plan acknowledged: (i) the need for augmentation of harm reduction interventions (principally injecting equipment provision (IEP)) to prevent HCV transmission and (ii) that monitoring of HCV transmission among PWID is essential to establish the impact of increases/changes in interventions.

Monitoring of HCV among PWID in Scotland and in other countries has usually involved measuring the prevalence of HCV antibodies - an indicator of past infection (Hutchinson et al., 2002; Roy et al., 2007). Although examining long-term trends in HCV prevalence can inform on the effectiveness of interventions, measures of incidence of $\mathrm{HCV}$ are much more useful in determining 
short term impact. The traditional method of measuring incidence - establishing seroconversion through follow-up and repeat testing of a cohort of individuals - can be expensive and logistically difficult to undertake. Few measures of HCV incidence among Scottish PWID have been generated to date, and most have used indirect methods (Roy et al., 2007).

Although harm reduction interventions for PWID - the foremost being opioid substitution therapy (OST) and IEP - have been shown to reduce self-reported injecting risk behaviour, there is a dearth of evidence with respect to their impact on HCV transmission in this population group (Palmateer et al., 2010). Rather than being an indication of the ineffectiveness of these interventions, it has been suggested that the lack of evidence may be due to limitations of studies that have been undertaken to date, such as employing ecological study designs and using crude measures of intervention uptake (for example, users vs. non-users of IEP services). Furthermore, few studies have measured the 'coverage' or intensity of interventions (e.g. the amount of injecting equipment distributed) (Lurie, 1997; Turner et al., 2011; Van Den Berg, Smit, Van Brussel, Coutinho, \& Prins, 2007).

In this paper, we investigate the individual-level associations between self-reported uptake of harm reduction interventions (IEP and OST) and incidence of HCV from a national survey of PWID carried out in Scotland. This study is the first large-scale, national application of a novel method designed to determine incidence of HCV using a cross-sectional design (Hope et al., 2011; Page-Shafer et al., 2008).

\section{Methods}

\section{Design and setting}

The Needle Exchange Surveillance Initiative (NESI) is a voluntary anonymous cross-sectional survey of PWID undertaken across mainland Scotland. Between June 2008 and June 2009, participants were recruited from 22 agencies and 81 pharmacies that provide sterile injecting equipment (these sites may also provide other harm reduction interventions, such as OST), which comprise $42 \%$ of IEP services in Scotland (ISD Scotland, 2010). Within logistical constraints (service manager agreement and a private room where the interviews could take place), services were selected to be broadly geographically representative. Eligible individuals had injected drugs in the past and had not participated in the study during the current survey year. Current injectors (defined as having injected in the last 6 months) were oversampled, if necessary, so that the proportion of the sample comprised by this group was at least $75 \%$ in each recruitment area. The study received ethical approval from the West of Scotland Research Ethics Service.

\section{Data collection}

Trained interviewers approached potential participants, explained the study and assessed them for eligibility and willingness to participate; reason for refusal, approximate age and gender of non-participants was recorded. After obtaining informed consent, the interviewers administered a questionnaire to establish information about participant demographics, injecting history, injecting risk behaviour, prison history and uptake of harm reduction interventions and other health services. Participants were also asked to provide a blood spot sample for HCV testing, which was obtained via fingerprick using a single-use disposable lancet. Individuals who completed the questionnaire were provided with a $£ 5$ voucher. All information collected was anonymous: a participant's dried blood spot (DBS) was linked to his/her questionnaire via a unique study number.

\section{Laboratory testing}

DBS were extracted and tested in a modification of the Ortho Save 3.0 EIA (Judd et al., 2003). Samples with optical densities of $<0.4,0.4-0.79$ and $\geq 0.8$ were classified as negative, weak reactive, and positive for HCV antibody (anti-HCV), respectively. HCV RNA testing was undertaken on anti-HCV negative samples using an 'in house' PCR assay: the bioMerieux extraction protocol for DBS on the Easymag and a real-time PCR (Bennett, Gunson, McAllister, Cameron, \& Carman, 2012). The assay detects to $1000 \mathrm{IU} / \mathrm{ml}$ in DBS. The testing was carried out in pools of five; samples in positive pools were then tested individually.

\section{Intervention measures}

The harm reduction interventions considered here are IEP and methadone maintenance therapy (MMT) (methadone is the most commonly prescribed opioid substitute in Scotland, accounting for $97 \%$ of the respondents who reported receiving pharmacological treatment for opioid addiction in the last 6 months in this study). Variables representing two types of IEP 'coverage' were generated: needle/syringe (N/S) coverage and non-N/S injecting equipment coverage were determined by dividing the reported number of obtained sterile N/S and non-N/S equipment (i.e. spoons, filters or water ampoules), respectively, by the self-reported number of injecting events in the previous six months. The distribution of the latter variables were examined and, given a very large proportion of individuals who reported receiving at least one N/S for every injection (i.e. 100\% coverage), it was suspected that respondents may have over-reported the numbers of N/S obtained. The threshold separating high and low N/S coverage was therefore set at 200\% (i.e. two needles/syringes for every injection). Since the survey contained no questions to ascertain methadone dosage, individuals were categorised according to whether they were currently receiving MMT, had received MMT in the last six months (but were not currently receiving it), or had not received MMT in the last six months. A combined measure of intervention coverage was created with categories low, medium and high: the combinations of $\mathrm{N} / \mathrm{S}$ coverage and MMT that were used to create these categories are listed in Box 1

. Respondents who were not receiving MMT and had not injected in the last six months were excluded.

\section{Statistical analysis}

Respondents who participated in the study more than once (duplicates) were identified in the database using initials, date of birth, gender and region of interview: either the first interview, or the interview with valid laboratory results, was retained for analysis. Out of a total of 2749 respondents, we identified 115 duplicates (Fig. 1). Anti-HCV weak reactives (representing only $2.7 \%$ of those with DBS results) were treated as anti-HCV positive.

In the very early stages of HCV infection, individuals have high levels of viraemia prior to developing antibodies (seroconverting); this has been referred to as the viraemic pre-seroconversion window period (Page-Shafer et al., 2008). Recent infections were therefore defined as individuals who were anti-HCV negative and positive for RNA on DBS testing. The following calculation was used to generate an estimate of incidence: $I=((365 / T) n) /((N-n)+(365 / T) n)$ where $I$ is the incidence, $T$ is the estimated duration of the viraemic pre-seroconversion window period, $n$ is the number of recent infections and $N$ is the number of susceptibles (i.e. anti-HCV negative individuals) (Hope et al., 2011). Estimates of the duration of the pre-seroconversion window period (28-65 days) were obtained from the published literature (Glynn et al., 2005; Netski et al., 2005; Page-Shafer et al., 2008). We did not 
Box 1: Categories of N/S coverage and MMT used to create combined intervention coverage.

\begin{tabular}{|c|c|c|c|}
\hline & \multicolumn{3}{|l|}{$\mathrm{N} / \mathrm{S}$ coverage } \\
\hline & $<200 \%$ & $\geq 200 \%$ & Did not inject in last six months \\
\hline \multicolumn{4}{|l|}{ MMT } \\
\hline Currently & Medium $(n=267)$ & High $(n=275)$ & High $(n=183)$ \\
\hline In the last six months (not currently) & $\operatorname{Low}(n=21)$ & Medium $(n=12)$ & $\operatorname{High}(n=0)$ \\
\hline Not in the last six months & Low $(n=218)$ & Medium $(n=140)$ & Excluded $(n=24)$ \\
\hline
\end{tabular}

calculate $95 \%$ confidence intervals around the incidence estimates as the uncertainty in the window period duration generated a wide range of potential values.

Logistic regression was undertaken to examine associations between recent HCV infection (i.e. anti-HCV negative and RNA positive) and self-reported uptake of harm reduction interventions (MMT and IEP). The reference group for comparison consisted of anti-HCV negative, RNA negative individuals. Associations between other variables and recent HCV infection were also explored. Univariable associations between each variable and recent infection were examined in turn. Multivariable models were subsequently built by including known or suspected confounders of the relationship between MMT/IEP and recent HCV infection; injecting risk behaviour variables and injecting frequency variables were not considered because they are on the causal pathway. Where there was a theoretical reason to suspect potential effect modification between two variables, the presence of interactions was assessed by entering interaction terms into the model individually and examining the $p$-values (based on the Wald test), as well as the effect sizes when the analysis was stratified by the effect modifier. Additionally, sensitivity analyses were undertaken to assess the robustness of the final model(s) to: (i) excluding unexpected results (incident infections who reported not injecting in the last six months) and (ii) restricting to current PWID (i.e. those who had injected in the last six months). All analyses were undertaken in SPSS version 14 .

\section{Results}

A total of 2629 respondents completed the questionnaire; comprising $63 \%$ of potentially eligible clients that were approached. Non-participants were slightly younger than participants (mean of 29 vs. 34 years); however, both participants and non-participants had the same gender distribution ( $72 \%$ male).

Among the 2555 respondents who provided a sufficient DBS, 1367 (54\%) were anti-HCV positive (including weak reactives) (Fig. 1). Twenty-four of the 1140 anti-HCV negatives (with sufficient sample) were found to be positive for HCV RNA. This generated incidence rate estimates ranging from 10.8 to 21.9 per 100 person years, corresponding to viraemic pre-seroconversion window period estimates of 28-65 days.

In univariable analyses (Table 1 ), the following variables were found to be significantly associated with increased odds of recent HCV infection: homelessness in the last six months,

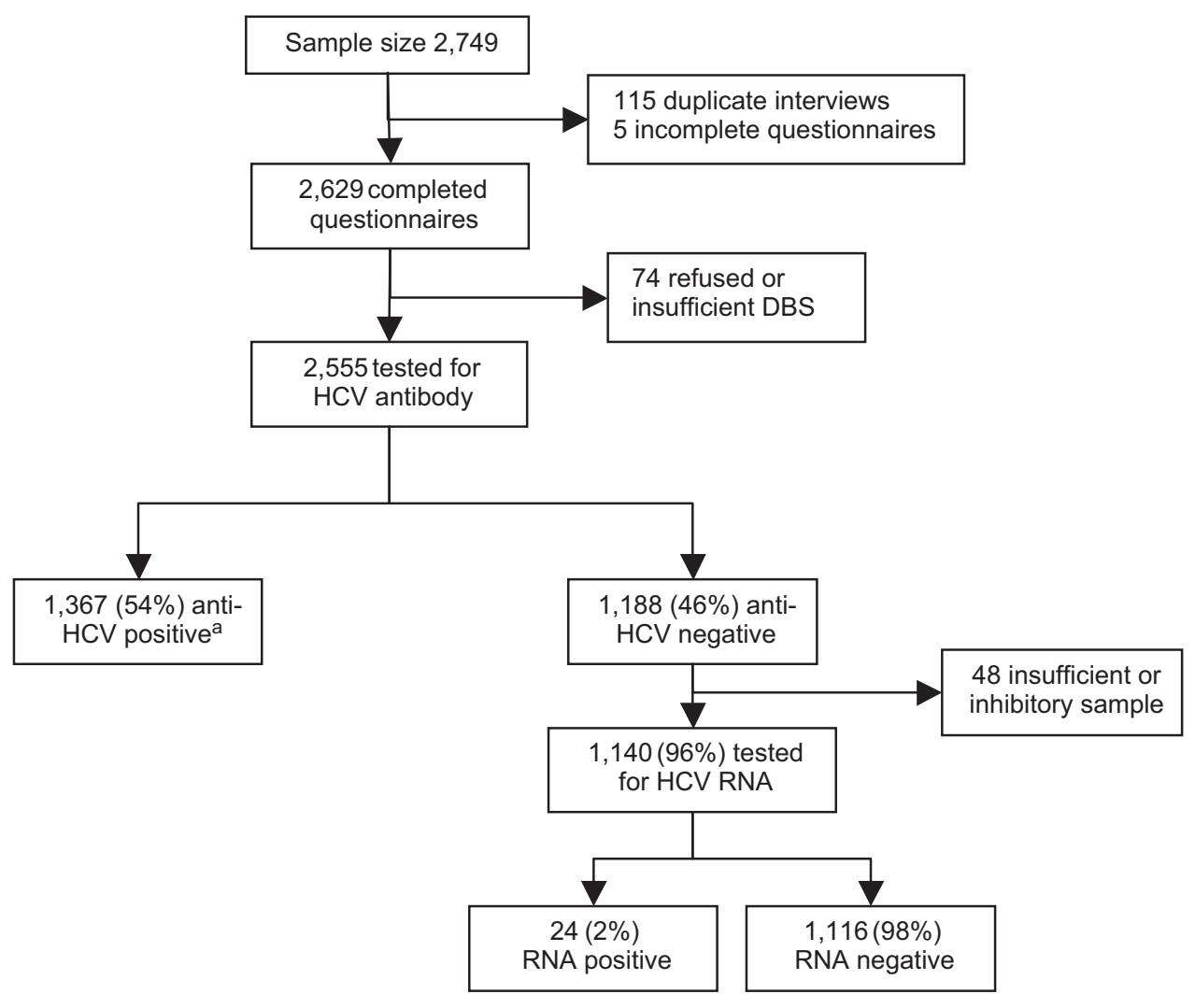

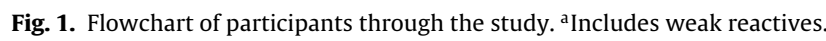


Table 1

Descriptive characteristics of study sample and univariable logistic regression analyses of associations with recent HCV infection $(n=1140)$.

\begin{tabular}{|c|c|c|c|c|c|c|}
\hline & No. HCV Ab-ves $(N)$ & No. recent infections ${ }^{\mathrm{a}}(n)$ & $\%(n / N)$ & OR & $95 \% \mathrm{CI}$ & $p$-Value \\
\hline \multicolumn{7}{|l|}{ Gender (5 non-responses) } \\
\hline Male & 835 & 17 & 2.0 & 1 & & \\
\hline Female & 300 & 7 & 2.3 & 1.15 & $0.47-2.80$ & 0.759 \\
\hline \multicolumn{7}{|l|}{ Age } \\
\hline $16-30$ & 573 & 17 & 3.0 & 1 & & \\
\hline$>30$ & 567 & 7 & 1.2 & 0.41 & $0.17-0.99$ & 0.048 \\
\hline \multicolumn{7}{|l|}{ Region } \\
\hline Greater Glasgow \& Clyde & 294 & 5 & 1.7 & 1 & & \\
\hline Elsewhere & 846 & 19 & 2.2 & 1.33 & $0.49-3.59$ & 0.576 \\
\hline \multicolumn{7}{|l|}{ Homeless in last 6 months } \\
\hline No & 886 & 10 & 1.1 & 1 & & \\
\hline Yes & 254 & 14 & 5.5 & 5.11 & $2.24-11.65$ & $<0.001$ \\
\hline \multicolumn{7}{|l|}{ Prison (2 non-responses) } \\
\hline Never & 641 & 9 & 1.4 & 1 & & \\
\hline In the last six months & 142 & 6 & 4.2 & 3.1 & $1.09-8.85$ & 0.035 \\
\hline In the past but not last six months & 355 & 9 & 2.5 & 1.83 & $0.72-4.64$ & 0.206 \\
\hline \multicolumn{7}{|c|}{ Excessive alcohol consumption (last 12 months) ${ }^{\mathrm{b}}$ ( 6 non-responses) } \\
\hline No & 863 & 11 & 1.3 & 1 & & \\
\hline Yes & 271 & 13 & 4.8 & 3.9 & $1.73-8.82$ & 0.001 \\
\hline \multicolumn{7}{|c|}{ Time since onset of injecting ( 2 non-responses) } \\
\hline$<5$ years & 447 & 14 & 3.1 & 1 & & \\
\hline$\geq 5$ years & 691 & 10 & 1.4 & 0.45 & $0.20-1.03$ & 0.059 \\
\hline \multicolumn{7}{|l|}{ Stimulant injection in last 6 months } \\
\hline No & 1008 & 19 & 1.9 & 1 & & \\
\hline Yes & 132 & 5 & 3.8 & 2.05 & $0.75-5.58$ & 0.161 \\
\hline \multicolumn{7}{|l|}{ Received MMT } \\
\hline In the last six months (not currently) & 33 & 3 & 9.1 & 1 & & \\
\hline Currently & 725 & 13 & 1.8 & 0.18 & $0.05-0.68$ & 0.011 \\
\hline Not in the last six months & 382 & 8 & 2.1 & 0.21 & $0.05-0.85$ & 0.028 \\
\hline \multicolumn{7}{|c|}{ Needle/syringe coverage (including N/S obtained from IEP services or other people) ${ }^{c, d}$} \\
\hline$<200 \%$ & 506 & 16 & 3.2 & 1 & & \\
\hline$\geq 200 \%$ & 427 & 4 & 0.9 & 0.29 & $0.10-0.87$ & 0.028 \\
\hline \multicolumn{7}{|c|}{ Combined intervention coverage (N/S and MMT) $)^{c, e}$} \\
\hline Low & 239 & 9 & 3.8 & 1 & & \\
\hline Medium & 419 & 8 & 1.9 & 0.5 & $0.19-1.31$ & 0.157 \\
\hline High & 458 & 6 & 1.3 & 0.34 & $0.12-0.97$ & 0.043 \\
\hline \multicolumn{7}{|l|}{ Filter coverage $e^{\mathrm{c}, \mathrm{d}}$} \\
\hline$<200 \%$ & 804 & 19 & 2.4 & 1 & & \\
\hline$\geq 200 \%$ & 129 & 1 & 0.8 & 0.35 & $0.05-2.65$ & 0.307 \\
\hline \multicolumn{7}{|l|}{ Spoon coverage $e^{\mathrm{c}, \mathrm{d}}$} \\
\hline$<200 \%$ & 811 & 19 & 2.3 & 1 & & \\
\hline$\geq 200 \%$ & 122 & 1 & 0.8 & 0.36 & $0.05-2.70$ & 0.318 \\
\hline
\end{tabular}

IEP, injecting equipment provision; MMT, methadone maintenance therapy; N/S, needles/syringes; OR, odds ratio.

a Anti-HCV negative and HCV-RNA positive individuals.

b As defined by UK Royal College of Physicians: >14 units/week for women and >21 units/week for men.

c See methods for definitions of coverage.

d Among individuals who reported injecting in the last 6 months.

e Excludes 24 respondents who reported not injecting and not receiving MMT in last six months.

having been in prison in the last six months, and excessive alcohol consumption. The following factors were significantly associated with reduced odds of recent HCV infection: age $>30$ years, receipt of prescribed MMT, $\geq 200 \% \mathrm{~N} / \mathrm{S}$ coverage (where sterile N/S had been obtained from IEP services or from other people), and high coverage of combined interventions. Longer time since onset of injecting ( $\geq 5$ years) was also associated with reduced risk and was marginally statistically significant $(p=0.059)$.

Table 2 presents two alternative adjusted models: (i) with MMT and IEP entered as separate variables in the model and (ii) with a combined measure of MMT and IEP. In model (i), those who had $\geq 200 \% \mathrm{~N} / \mathrm{S}$ coverage had a nearly $70 \%$ reduction in odds of recent HCV (AOR 0.3, 95\% CI 0.1-1.0), relative to those with $<200 \%$ coverage, after adjustment for region, gender, homelessness, imprisonment, time since onset of injecting and excessive alcohol consumption. The results were also suggestive of reduction in risk among both those on MMT currently and not on MMT in the last six months, relative to the baseline group of those who had been on MMT in the last six months, although neither association was statistically significant.
In model (ii), the reduced risk of recent infection among those with high coverage, as compared with low coverage, observed in univariable analysis (OR $0.3,95 \%$ CI $0.1-1.0$ ) was no longer statistically significant after adjustment (AOR 0.5, 95\% CI 0.2-1.5, $p=0.203$ ).

Suspected interactions between variables were investigated and there was some evidence that geographical region modifies the effect of MMT. Table 3 shows the adjusted odds ratios for MMT, stratified by region: in Greater Glasgow \& Clyde $(G G \& C)$ there was a marginally significant ( $p=0.055$ ) reduced odds of recent HCV infection among those currently on MMT, relative to those who had been prescribed MMT in the last six months but not currently (AOR 0.04, 95\% CI 0.001-1.1). This association was not seen in other Scottish regions.

Sensitivity analyses were undertaken to examine the robustness of the main effects model (Table 2i), as described in the methods. The effect sizes were robust to the exclusion of those who had not injected in the last six months and the four incident infections who had not injected in the last six months (data available from the authors on request). There was a loss of precision; however, this was expected due to the corresponding reduction in sample size. 
Table 2

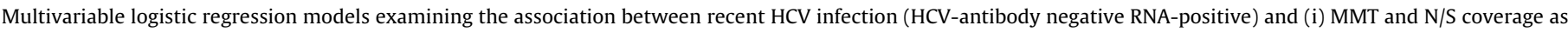
separate variables and (ii) combined MMT and N/S coverage.

\begin{tabular}{|c|c|c|c|c|c|c|c|}
\hline & & \multicolumn{3}{|c|}{ Univariable } & \multicolumn{3}{|c|}{ Multivariable $^{\mathrm{a}}$} \\
\hline & & OR & $95 \% \mathrm{CI}$ & $p$-Value & AOR & $95 \% \mathrm{CI}$ & $p$-Value \\
\hline \multirow{4}{*}{$\begin{array}{l}\text { Model (i) } \\
\text { Received MMT }\end{array}$} & & & & & $(n=1131)$ & & \\
\hline & In the last six months (not currently) & 1 & & & 1 & & \\
\hline & Currently & 0.18 & $0.05-0.68$ & 0.011 & 0.29 & $0.07-1.19$ & 0.086 \\
\hline & Not in the last six months & 0.21 & $0.05-0.85$ & 0.028 & 0.28 & $0.06-1.22$ & 0.089 \\
\hline N/S coverage (including N/S & $<200 \%$ & 1 & & & 1 & & \\
\hline obtained from IEP services or other & $\geq 200 \%$ & 0.29 & $0.10-0.87$ & 0.028 & 0.32 & $0.10-1.00$ & 0.05 \\
\hline people $)^{b}$ & Did not inject in last 6 months & 0.6 & $0.20-1.83$ & 0.372 & 1.3 & $0.38-4.43$ & 0.674 \\
\hline \multicolumn{5}{|l|}{ Model (ii) } & $(n=1107)$ & & \\
\hline Combined intervention coverage & Low & 1 & & & 1 & & \\
\hline \multirow[t]{2}{*}{$(\mathrm{N} / \mathrm{S} \text { coverage and MMT })^{\mathrm{b}}$} & Medium & 0.5 & $0.19-1.31$ & 0.157 & 0.5 & $0.18-1.35$ & 0.17 \\
\hline & High & 0.34 & $0.12-0.97$ & 0.043 & 0.48 & $0.16-1.48$ & 0.203 \\
\hline
\end{tabular}

(A)OR, (adjusted) odds ratio; IEP, injecting equipment provision; MMT, methadone maintenance therapy; N/S, needles/syringes.

a Adjusted for region, gender, homelessness, imprisonment, time since onset of injecting, and excessive alcohol consumption.

b See methods for definitions of coverage.

\section{Discussion}

We estimated an HCV incidence rate of $11-22$ per 100 personyears in this population of Scottish PWID. This is higher than a recently reported incidence rate of 5.9 per 100 person-years among a cohort of PWID in Wales (Craine et al., 2009) but lower than rates (38-47 per 100 person-years) reported in England (Hope et al., 2011; Judd et al., 2005). These variations may be attributable to differences in risk behaviour among regional injecting populations and/or study designs/recruitment approaches - two of the latter studies employed prospective cohort designs, and one recruited participants using respondent-driven sampling (RDS). Notably, the RDS study may have overestimated HCV incidence due to detection of a transmission cluster; the authors note that exclusion of this cluster would result in an incidence rate of $18-25$ per 100 person-years (Hope et al., 2011). Historically, regional incidence rate estimates among PWID in Scotland have ranged from 10 to 29 per 100 person-years (McDonald et al., 2012; Roy et al., 2007). However, whereas the latter estimates were generally confined geographically, our estimate applies to mainland Scotland.

A subset (Greater Glasgow \& Clyde region) of the data presented here was included in a previous analysis that examined the effect of harm reduction interventions by pooling data from UK studies (Turner et al., 2011). Turner et al. demonstrated an independent effect of N/S provision on incident HCV infection, a finding for which our study provides further evidence using data collected from across mainland Scotland. These studies are the first to observe a significant independent association between N/S provision and recent HCV infection (European Centre for Disease Prevention \& Control, 2011; Palmateer et al., 2010); possible reasons for this are likely to be the larger sample sizes (for example, in comparison to Van Den Berg et al. (2007)) and the use of more sensitive measures of exposure to N/S provision. Since crosssectional studies are generally cheaper and easier to undertake than prospective cohort studies, our study was able to achieve a large sample size through the application of a method to determine incidence using a cross-sectional design (Hope et al., 2011). Our study is also the first, to our knowledge, to look at sterile needles/syringes obtained from both IEP services and from other people, which may have further increased the sensitivity of this measure of exposure (since people who obtain sufficient sterile needles/syringes from others may have otherwise been misclassified as having low coverage and resulted in a dilution of the exposure-outcome association). This is an important finding that suggests secondary distribution may play a role in preventing HCV transmission (Bryant \& Hopwood, 2009; Lenton, Bevan, \& Lamond, 2006).

In contrast to Turner et al., we did not find a significant independent effect of MMT, nor a significant combined intervention effect, when considering Scotland overall. This could be due to statistical power: although we had a larger sample size (by approximately 200), we had fewer recent infections in our sample (24 vs. 40). Nevertheless, as demonstrated by Turner et al. and confirmed in our analysis (although we used a different measure of MMT), current MMT was associated with reduced odds of recent HCV infection in the Greater Glasgow \& Clyde region. Our finding that the reduced odds of those currently receiving MMT, when compared to those who had received MMT in the last six months (but not currently), suggests that individuals coming off opioid substitution are at increased risk of HCV infection. This pattern was not seen

Table 3

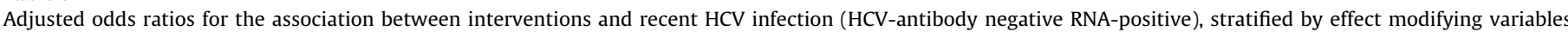
$(n=1131)$.

\begin{tabular}{|c|c|c|c|c|c|c|c|}
\hline & & \multirow[t]{2}{*}{$N$} & \multirow[t]{2}{*}{ Recent HCV $(n)$} & \multirow[t]{2}{*}{ Recent HCV (\%) } & \multicolumn{3}{|c|}{ Multivariable $^{\mathrm{a}}$} \\
\hline & & & & & AOR & $95 \% \mathrm{CI}$ & $p$-Value \\
\hline \multicolumn{8}{|c|}{ Prescribed MMT stratified by Scottish region } \\
\hline \multirow[t]{3}{*}{ GG\&C } & Last six months & 9 & 2 & 22.2 & 1 & & \\
\hline & Currently & 205 & 1 & 0.5 & 0.04 & $0.001-1.07$ & 0.055 \\
\hline & Not in the last six months & 77 & 2 & 2.6 & 0.16 & $0.01-2.55$ & 0.193 \\
\hline \multirow[t]{3}{*}{ Other Scottish regions } & Last six months & 24 & 1 & 4.2 & 1 & & \\
\hline & Currently & 515 & 12 & 2.3 & 0.73 & $0.08-6.64$ & 0.777 \\
\hline & Not in the last six months & 301 & 6 & 2.0 & 0.59 & $0.06-5.67$ & 0.647 \\
\hline
\end{tabular}

AOR, adjusted odds ratio; GG\&C, Greater Glasgow \& Clyde; MMT, methadone maintenance therapy.

a Adjusted for gender, homelessness, imprisonment, time since onset of injecting, and excessive alcohol consumption. 
in the other Scottish regions covered by this study: regional variations in the effectiveness of MMT may reflect differences in local delivery policies and practices. However, it is of note that our study applies the same design and questionnaire across all areas, whereas the Turner et al. study pooled data from studies which employed a mixture of different designs (respondent-driven sampling, cohort, cross-sectional) and questionnaires.

We did not find any significant associations between the provision of filters or spoons and recent HCV infection: again, this is possibly attributable to insufficient statistical power. Additionally, this study was undertaken prior to the substantial changes in IEP services associated with the Hepatitis C Action Plan in Scotland, one of which was an approximately five-fold increase in the provision of filters and spoons between 2008/09 and 2009/10 (ISD Scotland, 2011).

Non-intervention variables that were found to be associated with recent $\mathrm{HCV}$ infection in these analyses (age, homelessness, imprisonment) are also consistent with previous studies (Champion et al., 2004; Craine et al., 2009; Maher et al., 2006; Thorpe et al., 2002). We are not aware of any studies that have found an association between alcohol consumption and recent/incident HCV infection; however, previous studies have suggested a link between alcohol consumption and injecting risk behaviour (Arasteh \& Des Jarlais, 2009; Sander, Cole, Ostrow, Mehta, \& Kirk, 2010; Vidal-Trécan et al., 1998). The finding that those with shorter injecting histories ( $<5$ years) had a higher odds of recent $\mathrm{HCV}$ infection suggests that interventions targeted at new initiates to injecting may be important in reducing HCV incidence.

This study has a number of limitations. Firstly, it was not specifically designed to examine MMT as an intervention. We did not collect information on methadone dosage from participants and, furthermore, methadone dosage remains a problematic measure of methadone coverage since an 'adequate' dosage can vary greatly from person to person.

Selection bias, which has been well documented in other studies of IEP programmes (Lurie, 1997; Palmateer et al., 2010), may be present here. Since recruitment was from sites that provide sterile injecting equipment (some of which will also dispense MMT) rather than dedicated drug treatment sites, we are more likely to have sampled individuals who were receiving both MMT and N/S. We will also have oversampled those on MMT who continue to inject, since we confined the proportion of non-current injectors (i.e. had not injected in the last 6 months) to $25 \%$ of the sample. We may thus have been more likely to sample individuals receiving inadequate methadone dosages, since such individuals are likely getting injecting equipment in order to "top up" with heroin; measures of MMT effectiveness may therefore be underestimated. Recruitment at solely IEP sites may also bias the sample away from high risk injectors who are not in contact with services. Previous community-wide surveys undertaken in Glasgow showed that $90 \%$ of PWID recruited from street sites had visited IEP services in the past six months (Taylor et al., 2000); however, exclusion of individuals not in contact with services may still lead to underestimation of the impact of interventions.

We observed four recent infections among those who reported not injecting in the last six months. Two of these four individuals reported receiving an HCV-positive result from a previous test. Possible reasons for this discrepancy could be false positive PCR results or false negative anti-HCV results. It is also plausible that the respondents were dishonest or incorrectly recalled their behaviour; however, we cannot conclusively discount other risk factors, such as tattooing, on which we did not collect information. Self-reported risk behaviour among PWID may be subject to social desirability or recall biases; however, it is unlikely to differ systematically between those who received/did not receive interventions or those with/without the outcome of interest. Respondents may have overestimated self-reported uptake of clean needles/syringes but the effect of this is likely to be non-differential. Thus, the 200\% threshold is not meaningful in itself, except as an indicator of those with a higher ratio of clean N/S to injections. However, we recognise that the appropriate ratio of sterile $\mathrm{N} / \mathrm{S}$ to injections is important for policy-makers and injecting equipment providers, and therefore further work is required to elucidate this ratio.

The approach to calculating incidence applied in this study is heavily dependent on accurate estimation of the duration of the pre-seroconversion window period, around which there is uncertainty (Glynn et al., 2005; Netski et al., 2005; Page-Shafer et al., 2008). We examined intervention uptake in the six months prior to interview: since this time frame is longer than the upper estimates of the duration of the window period, this may contribute to some inaccuracy in the results.

The laboratory test to detect antibodies to HCV on DBS has been validated and has very high sensitivity and specificity (99\% and $100 \%$, respectively) (Judd et al., 2003); the respective values for the PCR test on DBS are $100 \%$ and 96\%, (Bennett et al., 2012). Recent infections could have been missed if the sample was taken during a 'dip' - a phase of undetectable viral load during a period of fluctuating viraemia that is observed, in some individuals, in the acute phase of HCV infection (Thomson, Smith, \& Klenerman, 2011). Conversely, a small proportion of the recent infections might have been false positives. There is also a chance that chronic infections could have been misclassified as recent infections in the case of immunosuppressed individuals (for example, those infected with HIV (Thomson et al., 2009)), who may have delayed seroconversion. Given the low HIV prevalence in the Scottish injecting population (Health Protection Scotland \& University of the West of Scotland, 2008), we do not believe this would be a significant factor; however, other lifestyle factors may contribute to immunosuppression, including the use of opiates themselves (Vallejo, de Leon-Casasola, \& Benyamin, 2004).

In conclusion, this study utilised a method of generating incidence using a cross-sectional design and demonstrated that high coverage of $\mathrm{N} / \mathrm{S}$ is associated with a reduction in recent $\mathrm{HCV}$ infection among PWID in Scotland. Despite the large sample size, statistical power was nevertheless an issue, since only 24 recent infections were detected among a sample of more than $1100 \mathrm{HCV}$ antibody-negatives. Additional sweeps of this survey will increase the cumulative sample size (and therefore power to detect associations) and allow us to examine the impact of the increase in interventions delivered by the Hepatitis C Action Plan for Scotland.

\section{Contributors}

E. Allen, A. Taylor, S. Hutchinson, N. Palmateer and D. Goldberg designed the survey. E. Allen and A. Taylor lead on the implementation of the survey. S. Cameron managed the laboratory testing of samples. N. Palmateer designed and lead on the statistical analysis, with support from S. Hutchinson. N. Palmateer wrote the first draft of the manuscript. All authors contributed to and have approved the final manuscript.

\section{Acknowledgements}

This work was supported by the Scottish Government. The authors are grateful to the following individuals and organisations: the respondents and services that participated in the NESI study; Cheryl Rees, Lauren Johnston, Julie Burza, Donald Falconer, Germana Vitrano and Alex Matheson for collecting the data; and Linda MacDonald, Tony Bimpson, Samantha Shepherd and Georgina McAllister at the West of Scotland Specialist Virology Centre for carrying out the testing of samples. 


\section{References}

Arasteh, K., \& Des Jarlais, D. C. (2009). At-risk drinking and injection and sexual risk behaviors of HIV-positive injection drug users entering drug treatment in New York City. Aids Patient Care and STDS, 23, 657-661.

Bennett, S., Gunson, R. N., McAllister, G. E., Cameron, S. O., \& Carman, W. F. (2012) Detection of hepatitis C virus RNA in dried blood spots. Journal of Clinical Virology, 54, 106-109.

Bryant, J., \& Hopwood, M. (2009). Secondary exchange of sterile injecting equipment in a high distribution environment: A mixed method analysis in south east Sydney, Australia. International Journal of Drug Policy, 20, 324-328.

Champion, J. K., Taylor, A., Hutchinson, S., Cameron, S., McMenamin, J., Mitchell, A., et al. (2004). Incidence of hepatitis $C$ virus infection and associated risk factors among Scottish prison inmates: A cohort study. American Journal of Epidemiology, 159, 514-519.

Craine, N., Hickman, M., Parry, J. V., Smith, J., Walker, A. M., Russell, D., et al. (2009). Incidence of hepatitis $C$ in drug injectors: The role of homelessness, opiate substitution treatment, equipment sharing, and community size. Epidemiology and Infection, 137, 1255-1265.

European Centre for Disease Prevention and Control. (2011). Evidence for the effectiveness of interventions to prevent infections among people who inject drugs. Part 1: Needle and syringe programmes and other interventions for preventing hepatitis C, HIV and injecting risk behaviour. Stockholm: ECDC.

Glynn, S. A., Wright, D. J., Kleinman, S. H., Hirschkorn, D., Tu, Y., Heldebrant, C., et al. (2005). Dynamics of viremia in early hepatitis C virus infection. Transfusion, 45, 994-1002.

Health Protection Scotland and University of the West of Scotland. (2008). The Needle Exchange Surveillance Initiative (NESI): Prevalence of HCV and injecting risk behaviours among injecting drug users attending needle exchanges in Scotland, 2007. Glasgow: Health Protection Scotland.

Hope, V. D., Hickman, M., Ngui, S. L., Jones, S., Telfer, M., Bizzarri, M., et al. (2011). Measuring the incidence, prevalence and genetic relatedness of hepatitis Cinfections among a community recruited sample of injecting drug users, using dried blood spots. Journal of Viral Hepatitis, 18, 262-270.

Hutchinson, S. J., McIntyre, P. G., Molyneaux, P., Cameron, S., Burns, S., Taylor, A., et al. (2002). Prevalence of hepatitis C among injectors in Scotland 1989-2000: Declining trends among young injectors halt in the late 1990s. Epidemiology and Infection, 128, 473-477.

ISD Scotland. (2010). Injecting Equipment Provision in Scotland Survey 2008/09. Edinburgh: NHS National Services Scotland.

ISD Scotland. (2011). Injecting Equipment Provision in Scotland Survey 2009/10. Edinburgh: NHS National Services Scotland.

Judd, A., Hickman, M., Jones, S., McDonald, T., Parry, J. V., Stimson, G. V., et al. (2005). Incidence of hepatitis $\mathrm{C}$ virus and HIV among new injecting drug users in London: Prospective cohort study. BMJ, 330, 24-25.

Judd, A., Parry, J., Hickman, M., McDonald, T., Jordan, L., Lewis, K., et al. (2003). Evaluation of a modified commercial assay in detecting antibody to hepatitis $C$ virus in oral fluids and dried blood spots. Journal of Medical Virology, 71, 49-55.

Lenton, S., Bevan, J., \& Lamond, T. (2006). Threat or opportunity? Secondary exchange in a setting with widespread availability of needles. Substance Use and Misuse, $41,845-864$.

Lurie, P. (1997). Invited commentary: Le mystere de Montreal. American Journal of Epidemiology, 146, 1003-1006.

Maher, L., Jalaludin, B., Chant, K. G., Jayasuriya, R., Sladden, T., Kaldor, J. M., et al. (2006). Incidence and risk factors for hepatitis $C$ seroconversion in injecting drug users in Australia. Addiction, 101, 1499-1508.
McDonald, S. A., Hutchinson, S. J., Cameron, S. O., Innes, H. A., McLeod, A., \& Goldberg, D. J. (2012). Examination of the risk of reinfection with hepatitis C among injecting drug users who have been tested in Glasgow. International Journal of Drug Policy, http://dx.doi.org/10.1016/j.drupo.2012.02.003.

Netski, D. M., Mosbruger, T., Depla, E., Maertens, G., Ray, S. C., Hamilton, R. G., et al. (2005). Humoral immune response in acute hepatitis $C$ virus infection. Clinical Infectious Diseases, 41, 667-675.

Page-Shafer, K., Pappalardo, B. L., Tobler, L. H., Phelps, B. H., Edlin, B. R., Moss, A. R., et al. (2008). Testing strategy to identify cases of acute hepatitis C virus (HCV) infection and to project HCV incidence rates. Journal of Clinical Microbiology, 46, 499-506.

Palmateer, N., Kimber, J., Hickman, M., Hutchinson, S., Rhodes, T., \& Goldberg, D. (2010). Evidence for the effectiveness of sterile injecting equipment provision in preventing hepatitis $C$ and human immunodeficiency virus transmission among injecting drug users: A review of reviews. Addiction, 105, 844-859.

Roy, K. M., Hutchinson, S. J., Wadd, S., Taylor, A., Cameron, S. O., Burns, S., et al (2007). Hepatitis C virus infection among injecting drug users in Scotland: A review of prevalence and incidence data and the methods used to generate them. Epidemiology and Infection, 135, 433-442.

Sander, P. M., Cole, S. R., Ostrow, D. G., Mehta, S. H., \& Kirk, G. D. (2010). Determinants of alcohol consumption in HIV-uninfected injection drug users. Drug and Alcohol Dependence, $111,173-176$.

Scottish Government. (2008). Hepatitis C action plan for Scotland, phase II (May 2008-March 2011). Edinburgh: Scottish Government.

Taylor, A., Goldberg, D., Hutchinson, S., Cameron, S., Gore, S. M., McMenamin, J., et al (2000). Prevalence of Hepatitis C virus infection among injecting drug users in Glasgow 1990-1996: Are current harm reduction strategies working? Journal of Infection, 40, 176-183.

Thomson, E. C., Nastoulia, E., Main, J., Karayiannis, P., Eliahoo, J., Muir, D., et al. (2009) Delayed anti-HCV antibody response in HIV-positive men acutely infected with HCV. AIDS, 23, 89-93.

Thomson, E. C. Smith, J. A., \& Klenerman, P. (2011). The natural history of early hepatitis $C$ virus evolution; lessons from a global outbreak in human immunodeficiency virus-1-infected individuals. Journal of General Virology, 92, 2227-2236.

Thorpe, L. E., Ouellet, L. J., Hershow, R., Bailey, S. L., Williams, I. T., Williamson, J., et al (2002). Risk of hepatitis $C$ virus infection among young adult injection drug users who share injection equipment. American Journal of Epidemiology, 155, 645-653.

Turner, K., Hutchinson, S., Vickerman, P., Hope, V., Craine, N., Palmateer, N., et al. (2011). The impact of needle and syringe provision and opiate substitution therapy on the incidence of Hepatitis C virus in injecting drug users: Pooling of UK evidence. Addiction, 106, 1978-1988.

University of the West of Scotland, Health Protection Scotland, \& West of Scotland Specialist Virology Centre. (2010). The Needle Exchange Surveillance Initiative (NESI): Prevalence of HCV and injecting risk behaviours among injecting drug users attending needle exchanges in Scotland, 2008/2009. Paisley: University of the West of Scotland.

Vallejo, R., de Leon-Casasola, O., \& Benyamin, R. (2004). Opioid therapy and immunosuppression: A review. American Journal of Therapeutics, 11, 354-365.

Van Den Berg, C., Smit, C., Van Brussel, G., Coutinho, R. \& Prins, M. (2007). Full participation in harm reduction programmes is associated with decreased risk for human immunodeficiency virus and hepatitis $C$ virus: Evidence from the Amsterdam Cohort Studies among drug users. Addiction, 102, 1454-1462.

Vidal-Trécan, G., Coste, J., Varescon-Pousson, I., Reboul-Marty, J., Christoforov, B., \& Boissonnas, A. (1998). Patterns of sexual and injecting risk behaviours in French intravenous drug users not reporting HIV and hepatitis $C$ virus seropositivities. Addiction, 93, 1657-1668. 